Posibilidades y limitaciones locales de la participación política juvenil en la red social Facebook. 


\title{
Posibilidades y limitaciones locales de la participación política juvenil en la red social Facebook ${ }^{1}$.
}

\section{Possibilities and limitations of local youth political participation in the social network Facebook.}

\author{
Carlos Alberto Durán Sánchez² \\ Universidad Santo Tomás, Bucaramanga, Colombia.
}

Artículo recibido en octubre de 2015; artículo aceptado en noviembre de 2015.

Citación del artículo: Durán, C. (2014). Posibilidades y limitaciones locales de la participación política juvenil en la red social Facebook. I+D Revista de Investigaciones, 6(2), 19-36.

\section{Resumen}

Este artículo pretende reflexionar sobre algunos aspectos que se requieren para analizar lo que está sucediendo en las redes sociales, especialmente para los jóvenes susceptibles de recibir y compartir información política, y con una alta probabilidad de desconexión con muchos de los discursos compartidos por candidatos y políticos, quiénes bien podrían tener una caracterización de Inmigrantes Digitales, sobre todo porque sus estrategias erradas y el desinterés en el tema, contribuyen y se materializan en la continuación de la alta abstención electoral.

Palabras claves: política, juventud, redes
Abstract

This article aims to reflect on some aspects required to analyze what is happening on social networks, especially for young people eligible for get and share information, and with a high probability of disconnection shared by many of the candidates and political speeches who may well have a characterization of Digital Immigrants, especially because their strategies wrong and disinterest in the subject, and contribute materialize in the continuation of the high voter turnout.

Keywords: politics, youth, social networks. sociales.

\footnotetext{
'Artículo de reflexión, enfoque cuantitativo, financiado por la Universidad Santo Tomás de la ciudad de Bucaramanga (Colombia). Dirección Carrera 18 No. 9 -27 Bucaramanga, PBX: 6800801. Fecha de inicio: enero de 2014, fecha de terminación: enero de 2015.

${ }^{2}$ Comunicador social, Universidad Autónoma de Bucaramanga. Magíster en Historia, Universidad Industrial de Santander. Docente-Investigador Universidad Santo Tomás de la ciudad de Bucaramanga (Colombia): Dirección: Carrera 18 No.9-27 Bucaramanga,PBX: 6800801.Correo electrónico: carlosaduran@yahoo.es
} 


\section{Introducción}

El uso del concepto "nativos digitales" es común para referirse a la diferencia generacional existente entre las personas nacidas en la era digital -entendida como la convergencia tecnológica contemporánea que ha llegado a reorganizar contenidos, modos de trabajo y de entretenimiento, usando productos electrónicos que requieren de usuarios supremamente activos- y las nacidos en las décadas de los años setenta y ochenta, en la llamada era electrónica, y que han tenido que migrar desde formas de interacción comunicativas analógicas tradicionales hacia otras mayormente cimentadas en nuevas tecnologías de interacción constante, con gran uso de redes globales y con personas que mantienen una presencia ausencia del "yo" bajo diferentes perfiles.

Cuando los jóvenes colombianos de hoy nacieron (entre 1988 y 1996) el uso de herramientas electrónicas que posibilitaban una mayor conectividad entre los seres humanos, como computadores personales y teléfonos móviles estaba imponiéndose, mientras que en los años anteriores vieron la luz ciudadanos que contemplaron las comunicaciones con tecnologías diferentes como el teléfono y el telegrama, y ante la llamada revolución microelectrónica (Mires, 2009), tuvieron que adaptarse rápidamente a los nuevos avances.

Son precisamente las redes sociales y su éxito vertiginoso, un fenómeno de amplio desarrollo y crecimiento que estaría acentuando la brecha generacional entre inmigrantes y nativos, porque podrían ser concebidas de manera diferente, especialmente en cuanto a su practicidad, ya que fueron aprendidas y comprendidas por los inmigrantes desde el modelo mental de su propia experiencia.

Los nativos digitales en Colombia votan muy poco, pese a que en las redes sociales, a menudo, son altamente participativos en temas políticos . Aunque la alta abstención electoral juvenil es un fenómeno común a la mayoría de países con democracia participativa, las nuevas tecnologías podrían posibilitar una mayor intervención democrática y deliberativa que quizá también podría traducirse en una mayor participación electoral.

Quiero expresar en las limitaciones, entendidas como las circunstancias que dificultan el hecho de compartir contenidos y la participación cívica y política de Facebook en el ámbito local, y que sin embargo podrían verse reflejadas en unos mayores niveles de participación electoral para los jóvenes. Asimismo, se expresará un punto de vista personal acerca de las posibilidades, entendidas como el conjunto de circunstancias y medios disponibles en la red social que pudieran ayudar a tener un mejor uso político por parte de políticos, campañas y los mismos usuarios.

\section{Las redes, novedosas y con audiencias que crecen y se desarrollan}

La sociedad, en términos generales, siempre ha mantenido círculos en los cuales los hombres se dividen y se integran de acuerdo a intereses comunes. Los clubes sociales, los grupos de apoyo o los entornos familiares constituyen redes con adeptos deliberativos, partícipes y con ánimo de construir mejores comunidades. La 
facilidad de las comunicaciones digitales y la irrupción masiva de internet en la última década del siglo XX revolucionaron la comunicación humana porque encontraron la plataforma perfecta para mayores integraciones comunes. Fernández (Flores, 2009), afirma que la importancia de las redes sociales no estriba en su simple uso para juegos y encuentros inesperados, sino en haberse constituido en un espacio virtual "para desarrollar proyectos, integrar comunidades, poner en pie servicios, tomar decisiones en tiempos complejos, y proyectarse hacia el mercado global usando toda la potencia de la virtualidad".

El término web 2.0 abrió paso a plataformas conocidas como redes sociales, que tienen como característica la unión de usuarios que se van agregando de acuerdo a intereses comunes y a cuestiones por compartir. Por ejemplo, no son iguales las plataformas académicas como ResearchGate o ResearchId a otras como Facebook, Twitter o Myspace, cuyas características generales difieren en la forma y en los propósitos para la interacción grupal, ya que estas últimas conservan un aspecto de autovisibilidad y de autorreconocimiento aún mayor, además de usuarios o audiencias diferentes.

La idea sobre el empoderamiento de las audiencias en medios digitales, que en estos años recientes están basadas más en el egocentrismo de poder escoger lo que se ve y lo que se escucha, que en la pasividad de los medios tradicionales, fue expresada por Alves (2014), director del Centro Knight para el periodismo en las Américas de la Universidad de Texas. Este concepto de empoderamiento bien podría servir de guía para entender comportamientos de los usuarios de las redes sociales, en cuanto a la decisión de compartir información en sus muros o líneas de tiempo.

La potestad para decidir a cuáles personas seguir, limitar, o permitirles el acceso a contenidos personales; la decisión de compartir entre amigos lejanos o cercanos, de eliminar o bloquear virtualmente a otros, de interactuar de acuerdo a lo que se desea mostrar de la propia persona, o lo que se quiere ver de otros sin que se den cuenta, son una nueva manera de comunicación en la que la retroalimentación ocupa un papel predominante y el poder de decisión cobra fuerza, igualmente.

No obstante, la decisión del vínculo constante en redes sociales es también enorme en las edades de los migrantes digitales en Colombia. Actualmente $5.163 .659(23.6 \%)$ ciudadanos colombianos son usuarios de Facebook, en edades comprendidas entre los 35 y los 80 años. Por su parte, los nativos digitales son 10.786.840 (49.3\%), en edades comprendidas entre los 13 y los 24 años. Esta duplicidad entre unos y otros usuarios con una brecha generacional podría tener características distintivas en cuanto a la manera en que se percibe la participación política en los muros.

Abstenerse de estos recursos y de estas herramientas con las que se cuenta en el mundo de hoy podría ser impensable para los jóvenes que han incorporado tanto procesos mentales atraídos por las multitareas, como la capacidad de recibir mucha información susceptible de ser compartida, procesada o dejada a un lado. Una de las características de los nacidos una generación 
anterior, los inmigrantes digitales, tiene que ver con prejuicios valorativos, que según Prensky (2010) estriban en una subvaloración de las habilidades de los nativos, de sus niveles de adaptabilidad, de aprendizaje y de perfeccionamiento luego de muchos años de acciones entre grupos y tras de numerosas prácticas comunes.

En ese mismo sentido, el uso tan frecuente de las redes sociales y de las herramientas tecnológicas por parte de los nativos tiene una explicación básica para los migrantes: tienden a pensar que los primeros mantienen una especie de esclavitud tecnológica. La contradicción a este punto de vista fue expresada por Castells (2009; ) y Fidalgo, Serrano, Cnhavilas, \& Correia (2013) al afirmar que no era cierta la esclavitud de la tecnología, sino que, por el contrario, existía una mayor libertad de uso del tiempo libre. Ciertamente, lo que ha cambiado es la forma de usarlo. De la misma manera, la sensación de privacidad en las redes sociales tiene un elemento falaz, porque en realidad todos nuestros datos y contenidos son manejados por plataformas disímiles y con fines que desconocemos. Al señalar "acepto" dichos contenidos son activados de manera integral.

Para los nativos digitales el uso de las redes no es intuitivo, pero, a medida que se desarrolla su permanencia en ellas van adquiriendo mayores conocimientos sobre su manejo y sus maneras de participar activamente. Las redes sociales pasan por un proceso de alfabetización mediática (Leonard, 2012) que origina una mayor participación. Este proceso también transforma su capacidad crítica.
Desde el compromiso político, es un desarrollo que depende de algunos factores como el económico porque facilita el acceso a las nuevas tecnologías, pero también de factores sociales y psicológicos que explican el crecimiento del conocimiento, tanto personal como grupal sobre estos temas. En ello se puede ver una relación basada en que el comportamiento electoral está fuertemente marcado por la definición propia y grupal del ciudadano, lo que piensa acerca de su rol en la sociedad, y la manera como deriva su comportamiento. Estos factores finalmente le aportan las condiciones para explicar la abstención, teniendo la edad como uno de sus elementos importantes. Milbrath \& Goel (Pérez, 2006) afirman que "no es la edad cronológica lo que importa sino la experiencia y la adquisición de responsabilidades que se relacionan con la edad".

En cuanto al ejercicio del poder en la red, Castells señala su funcionamiento variable en el tiempo de acuerdo con las variaciones de las plataformas, la aparición de otras formas de funcionamiento y también de la capacidad de los usuarios como actores sociales para modificar su funcionalidad según sus propios intereses (2009). "La sociedad red global es una estructura dinámica, altamente maleable a las fuerzas sociales, la cultura, la política y las estrategias económicas".

Señala también que el poder de influenciar en los comportamientos de otras personas requiere de dos mecanismos básicos. Por una parte, el de programar o reprogramar las redes según diferentes objetivos, y por otra, el de conectar 
otras redes de manera estratégica, tal como sucede con los medios de comunicación tradicionales, que generan contenido en la red, al mismo tiempo que blogs, Facebook y Twitter muestran contenidos favorables o llamativos hacia el candidato con el propósito de evitar la competencia de otras redes.

En cuanto a la participación política, algunas investigaciones (Brader, 2006; Westen, 2007), han encontrado que las personas tienen la tendencia a continuar creyendo en sus propias convicciones políticas, siendo motivadas por elementos como el entusiasmo y el miedo, desde el apoyo al candidato de preferencia o al miedo de votar por el contrario. Sería muy interesante observar estas dos motivaciones en las últimas elecciones presidenciales en Colombia para entender cuántas personas votaron por Juan Manuel Santos porque tenían miedo al otro candidato, y viceversa. Hacer surgir un miedo aún mayor a la simple animadversión natural hacia el candidato que no es de la preferencia, puede ayudar también a conseguir nuevos votantes, especialmente cuando son presentadas las posibles consecuencias negativas en el caso de la victoria de uno u otro.

Precisamente, Brader encuentra en su investigación "que no había oposición entre los asuntos emocionales y los racionales. Las emociones confieren fuerza al argumento" (2006), de tal manera que los jóvenes podrían ser motivados en Facebook a votar por los candidatos que se adecúen a sus sentimientos y emociones, más que por los que presenten mejores argumentos. Este factor puede ser muy importante para entender su comportamiento.
De igual forma, resulta oportuno traer a colación dos ejemplos importantes sobre el uso de las redes sociales en la promoción de la participación política. El primer caso se desarrolló a través de Facebook y tuvo repercusiones mundiales. La marcha llamada "Un millón de voces contra las Farc", cuyo eslogan "No más Farc" logró canalizar a través de Facebook una oleada de descontento enorme en la ciudadanía que protestó por los secuestros y asesinatos del movimiento armado ilegal. Según fuentes del mismo grupo en la red social y de medios de comunicación como El Tiempo, lograron reunir 250.000 usuarios en la red, y posteriormente, el 4 de febrero de 2008, sin tener datos más precisos, se movilizaron alrededor de seis millones de personas en 193 ciudades de Colombia y del mundo. Esta capacidad de convocatoria correspondió a comités de usuarios bien organizados y con labores específicas que contaron con un momento coyuntural.

Otro caso a tener en cuenta sucedió en Bogotá, cuando se llevaba a cabo la campaña electoral a la alcaldía en el año 2011. Jaime Castro, quien fuera alcalde en 1992, compitió nuevamente frente a Gustavo Petro, Enrique Peñalosa, Gina Parody, y ocho candidatos más. Un especialista en mercadeo político en redes sociales contratado en su campaña, aconsejó adelantar una estratégica ofensiva en blogs y redes sociales, y luego de hacer un filtro de posibles votantes, entre quienes se encontraban los que opinaban y participaban favorablemente por el candidato, decidieron invitar un número de mil simpatizantes a su sede política. La sorpresa que se llevaron cuando solamente una persona acudió a la cita (Dallmeier, 2014), fue enorme. Posteriormente, 
el candidato fue el último de los nueve, obteniendo tan solo 10509 votos $(0.46 \%)$ comparado con el ganador, Gustavo Petro, que logró $721.508(32.16 \%)$.

\section{¿Por qué los jóvenes, por qué la tecnología y por qué la política?}

Los filósofos de la llamada Teoría Crítica, como Horkheimer y Marcuse, habían expresado tempranamente una preocupación frente al hecho de que el desarrollo de objetivos basados en un raciocinio totalitario de la sociedad industrial convirtió al hombre en un ser unidimensional, cuyo predominio de lo utilitario llevó a una transformación y a una reducción de la sociedad en industria, y transformó a los hombres en autómatas para quienes la ciencia y su desarrollo no estaban al servicio del mejoramiento de la existencia humana en su totalidad, sino al servicio del mantenimiento de una civilización productiva.

Fue Habermas (1999), quien se interesó en lograr una teoría social capaz de orientar en la práctica lo que es correcto y lo que no, y capaz de generar cambios en lo colectivo, especialmente con el establecimiento de conceptos sobre las acciones comunicativas, cuyo estudio es básico para el entendimiento de la vida social política con un propósito emancipatorio y crítico en la llamada modernidad. Desde este punto de vista los actos comunicativos en el interior de las redes sociales como fenómeno tecnológico, tienen, además de la movilidad como característica acrecentada por la posibilidad de hacer uso de teléfonos móviles y tabletas con plataformas rápidas, la del manejo de una autonomía en la interacción, de acuerdo a múltiples factores que varían según los contextos socio - culturales, y que en la práctica podrían ser puestos para generar cambios en lo colectivo, para mejorar la manera en que la participación política gesta un cambio en la nación colombiana. Esta transformación requiere de una mayor participación política juvenil.

Ahora bien, la población joven en Colombia, con edades comprendidas entre los 18 y los 29 años, podría decidir en forma significativa los resultados, ya que conserva el $46 \%$ del potencial electoral, un factor estadísticamente definitorio para cualquier candidato. Sin embargo, los jóvenes votan menos en la mayoría de democracias por varios factores.

En primer lugar, desde el enfoque sociopsicológico de la ciencia política, los jóvenes votan menos porque su identificación hacia partidos políticos no está consolidada como en los votantes mayores; igualmente, porque su desconocimiento de temas políticos va de la mano con un sentimiento de inconformidad. El Registrador Nacional del Estado Civil, Carlos Ariel Sánchez (2013), afirma que las razones por las cuales solo un $10 \%$ del potencial electoral de los jóvenes en edades comprendidas entre los 18 y los 24 años vota - una cifra baja relacionada con el $50 \%$ de los mayores de 40 años-podrían deberse "a factores de conocimiento de una parte, y de otra, a su grado de confianza en las instituciones. La gente joven, generalmente es rebelde y contestataria, y puede expresar la inconformidad con la abstención".

En segundo lugar, desde el enfoque de la decisión racional, porque los beneficios que obtienen como ciudadanos, tales como el económico o las gestiones de los gobiernos, son 
pensados y asumidos desde el corto plazo. Este factor podría verse extendido ante las posibilidades de las nuevas tecnologías.

Encontrar si existe un factor diferenciador de la participación política entre quienes son usuarios de internet y quienes no lo son, ha sido un tema de reflexión temprana, toda vez que existía una posibilidad abierta de constituir un recurso valioso para el ejercicio de la ciudadanía. Por ejemplo, mediante un análisis descriptivo de perfiles concretos y un análisis bivariable de las prácticas políticas en España, se encontró que los internautas frecuentes tienen un sentimiento desfavorable hacia la política, mayor a quienes no son usuarios asiduos de la red. Sin embargo, existe también una valoración superior hacia la interacción con los políticos, especialmente porque consideran que las opiniones expresadas en internet pueden ser escuchadas. Esta circunstancia ya indicaba que se avecinaba un cambio en la cultura política para los usuarios de internet, no porque se hubieran notado cambios sociales, sino porque las mismas necesidades de aquellos cambios tienen ahora mayores posibilidades de ser expresados. Igualmente, la abstención del voto real de los internautas, según el estudio, fue tres veces más alta al ser comparados con los no usuarios de internet, pese a que valoraban enormemente la idea de participación democrática. La explicación en España estaría dada porque: "El apoyo a la idea es grande, pero la decepción se acrecienta en cuanto se plasma en un estado concreto, organizaciones o instituciones concretas y finalmente personas que ejercen una profesión concreta, la de político"(Mayo, 2005).

Ahora bien, el uso de herramientas digitales para promover la participación personal se desarrolló más allá de las fronteras de los computadores de escritorio, encontrando en la comunicación móvil toda una posibilidad real en la política. En cuanto a la tecnología combinada como herramienta política, Castells (2009) explica que existen dos características principales en la comunicación móvil: la autonomía y los espacios de flujos de tiempo intemporal. En el primer caso, porque la gente cada vez es más autónoma para trabajar en conjunto, y en el segundo, porque no es necesaria una continuidad en el tiempo para desarrollar múltiples tareas y operaciones.

No en vano, desde hace unos años, se ha venido discutiendo en España, con el movimiento 15M, también llamado "Los indignados", la posibilidad de que los ciudadanos puedan ejercer el voto de manera virtual, lo cual, aunque tiene riesgos, también supondría toda una revolución para las campañas, para los candidatos, para la comodidad del ciudadano, pero también para la participación democrática. En este sentido, en el año 2011 se llevó a cabo una experiencia real en las elecciones de Estonia contabilizando los votos realizados a través de internet en unas elecciones presidenciales. Para ello era necesario validar el voto por medio de una tarjeta de firma electrónica y dos códigos pin. La participación aumentó en un $15.4 \%$ de la votación (Duch, 2011).

No obstante, hablar de excesivos beneficios en el uso de las redes sociales para la participación cívico-política podría tener riesgos y acepciones simplistas, porque también podría pensarse que en las relaciones entre los miembros de las redes se pueden establecer prácticas menos 
participativas, autoritarias o tendenciosas, tal como lo señalaron Buckingham \& Rodríguez (2013) en un reciente estudio sobre un mundo virtual para jóvenes, llamado Habbo Hotel, que se promocionaba como "democrático".

Igualmente, el ejercicio electoral a través de internet podría tener complicaciones, ya que si bien mantiene el altruismo de una mayor participación que legitimaría las decisiones políticas, requiere una mayor profundización en cuanto a la información ciudadana, la promoción de cultura política y los mecanismos efectivos de procesos y control (Reniun, 2008).

$\mathrm{Si}$ bien es cierto que algunos activismos políticos exitosos de las comunicaciones en Facebook, como el de Egipto y la llamada Primavera Árabe, podrían dar cuenta que el ejercicio de una ciudadanía digital participativa se está convirtiendo en una herramienta para "mover el mundo" (Orihuela, 2008), algunos estudios han revelado que la participación política es escasa en ir más allá del mundo virtual, y que por el contrario inicia y termina en él (García, Del Hoyo, \& Fernández, 2014).

También son conocidos algunos de los factores participativos antidemocráticos, como cerrarse al activismo, el bloqueo de opiniones, los insultos, y las burlas despiadadas. A pesar de que los mismos usuarios hacen llamados al activismo social cuando se trata de injusticias, causas ambientalistas o situaciones en las que se requiera la solidaridad, la libertad y el anonimato en las redes sociales también posibilitan los mensajes abusivos, injustos y totalitarios.

La interacción social en redes como Facebook es parecida a la vida real en cuanto a que solo en ocasiones logra mover a los usuarios y amigos hacia la participación real, alejándose de los conceptos iniciales que preveían, a inicios del siglo XXI, un diálogo más estrecho entre la ciudadanía y el poder político.

Un informe reciente del Pew Research Center encontró que en India, los ciudadanos se muestran poco interesados en compartir contenido político en Facebook. Aunque el acceso a internet en este país es muy bajo, solo el $16 \%$ de la población de 1200 millones de personas, es decir, aproximadamente 192 millones de ciudadanos. De ellos, solo el $51 \%$ reconoce usar redes sociales, aunque reportes de páginas especializadas como owloo.com dicen que en este país actualmente hay 112 millones de usuarios de Facebook (que ajustado correspondería al $58.4 \%$ ). Sin embargo, los usuarios de redes sociales reconocen que en un $90 \%$ las usan para mantenerse en contacto con familiares y amigos, y solo un 35\% para ver o compartir sobre asuntos políticos (Poushter, 2014).

Este mismo centro de investigaciones afirma que ha encontrado actitudes y patrones similares en otros países en desarrollo. Probablemente obedezcan a contextos endémicos y a si las relaciones se pueden convertir en movilizadoras de acuerdo al mensaje, cuyo realce pareciera ser supremamente importante para la acción y la comunicación de la información.

Un análisis reciente sobre las marchas estudiantiles chilenas del 2011 (Cabalin, 2014) reveló que el $26 \%$ del contenido publicado en el perfil de Facebook de la Federación Estudiantil 
Chilena $(\mathrm{FECH})$ provino de otros medios ajenos a la propia organización, indicando que la página corresponde mayoritariamente a la réplica de otros contenidos incluidos en medios tradicionales $\mathrm{u}$ otros portales, el movimiento combinó un efectivo trabajo en varios lugares de diferentes ciudades, con actividades singulares. Siendo una mezcla de "Facebook y calle".

Figura 1. Ejemplos de usuarios de Facebook en el año 2014 de acuerdo al número de habitantes en India, Colombia y el municipio de Bucaramanga.

INDIA

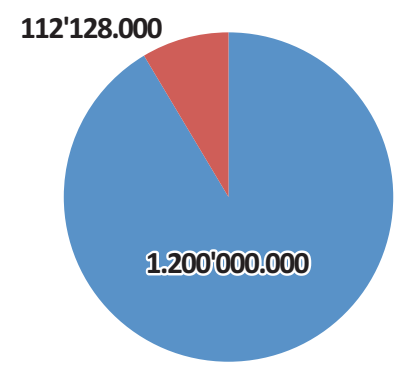

COLOMBIA

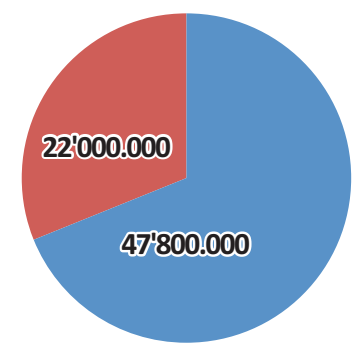

BUCARAMANGA

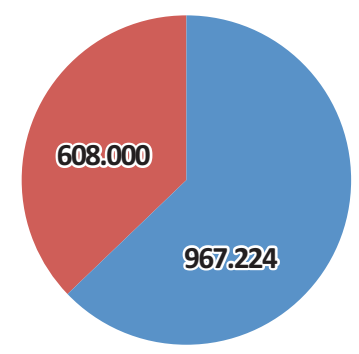

Fuente: El autor.

Aceptación de contenidos y de políticos en Facebook Colombia

Algunos datos que tienen que ver con la visibilidad de perfiles políticos en Facebook permiten distinguir algunos factores interesantes. Un ejemplo es el del político dominicano Tito Hernández, que tiene 14.081 seguidores de Facebook en Colombia, siendo el tercer país en América Latina, luego de México y de su propio país, donde mantiene 71.837 seguidores. Su perfil es definido como el de una figura pública que analiza la actualidad política dominicana y del mundo, promoviendo la toma de conciencia a través de sus publicaciones. Igualmente sucede con Josefina Vásquez Mota, una diputada, economista y escritora mexicana, excandidata presidencial por el partido PAN en el 2013, que se autodefine como una ciudadana que lucha por la construcción de un México diferente, libre y democrático. En México tiene 1.729.154 seguidores. Colombia es su cuarto país con 2230 seguidores, luego de Estados Unidos y Argentina.

Aunque los políticos extranjeros con más seguidores en Colombia son el presidente de Estados Unidos, Barack Obama (209.353) y los venezolanos Henrique Capriles (77.309) y Leopoldo López (36 047), cuyas razones de aprecio podrían explicarse por la popularidad mundial el uno, o por la solidaridad política de los colombianos los otros dos, también llama la atención que los seguidores del dominicano y la mexicana son altos si se compara con la mayoría de congresistas colombianos.

Pocos son los políticos nacionales que presentan estas características de popularidad. Además del presidente Santos (727.404), del expresidente Uribe (462.333), y de los excandidatos presidenciales recientes, solo un puñado de congresistas tiene un alto número de 
seguidores en Facebook. Ellos son: Clara López (197.083), Jorge Enrique Robledo (135.379) y David Luna (37.453). A nivel local quién más seguidores tiene en Facebook es el gobernador Richard Aguilar con 19.604, seguido de la congresista María Eugenia Triana con 4981. Por su parte, políticos nacionales reconocidos como Antonio Navarro con 1703, o locales con bastante experiencia como Jaime Durán Barrera con 201 , tienen un bajo número de seguidores en dicha red social.

Un reciente análisis sobre la percepción política en Bucaramanga realizado por Álvarez, Granados \& Hernández (2013), al correlacionar variables, encontró que en los jóvenes universitarios en la ciudad existe una actitud menos favorable hacia la democracia y poca comprensión política, porque encuentran que la información es confusa. Sin embargo, también expresan un alto optimismo hacia los procesos democráticos, y una alta valoración de los líderes, los partidos, la participación electoral y la eficacia de la política. Algo así como "no nos gusta la democracia actual, pero somos optimistas en que va a mejorar y en que los líderes lo van a hacer mejor".

Vale la pena resaltar que la edad guarda una correlación directa con las actitudes políticas, y aunque débil, es posible observar que a mayor edad, mayor satisfacción por la democracia; pero también a mayor edad la actitud hacia la política es menor. A nuestro parecer, las ambigüedades e indefiniciones de parte de los estudiantes encuestados podrían deberse a un nivel de desinformación originado por un alto nivel de confusión, que reconocieron tener, sobre las informaciones políticas.

Precisamente la red social Facebook ha sido usada, especialmente en la última campaña presidencial, como una plataforma para desinformar o subinformar por parte de opositores políticos que contó con todo un diseño estratégico de guerra sucia, y que tuvo como consecuencia una mayor actividad dentro de los muros popularizando los contenidos y que conllevó incluso a acciones penales.

De hecho, las actividades políticas en Facebook son extendidas cuando ciertos tópicos y mensajes se vuelven populares. Por ejemplo, las críticas a las actuaciones del Congreso colombiano, las campañas de solidaridad contra el maltrato animal, la preservación del medio ambiente contra la minería, o las pasiones suscitadas a favor o en contra del expresidente Álvaro Uribe Vélez. Es decir, si se trata de participación política habría que medir la efectividad de la interacción juvenil con estos temas, y si ellos se materializan como participación electoral.

El ejercicio del voto real en urna difiere de la participación cívica política. En ello parece existir una dicotomía entre la intervención virtual y la acción fuera de ella, toda vez que la primera se mantiene muy activa y la segunda no. 
Figura 2. Políticos colombianos (color amarillo), extranjeros (color azul), y santandereanos (color verde) de acuerdo al número de seguidores en Facebook desde perfiles ubicados en Colombia.

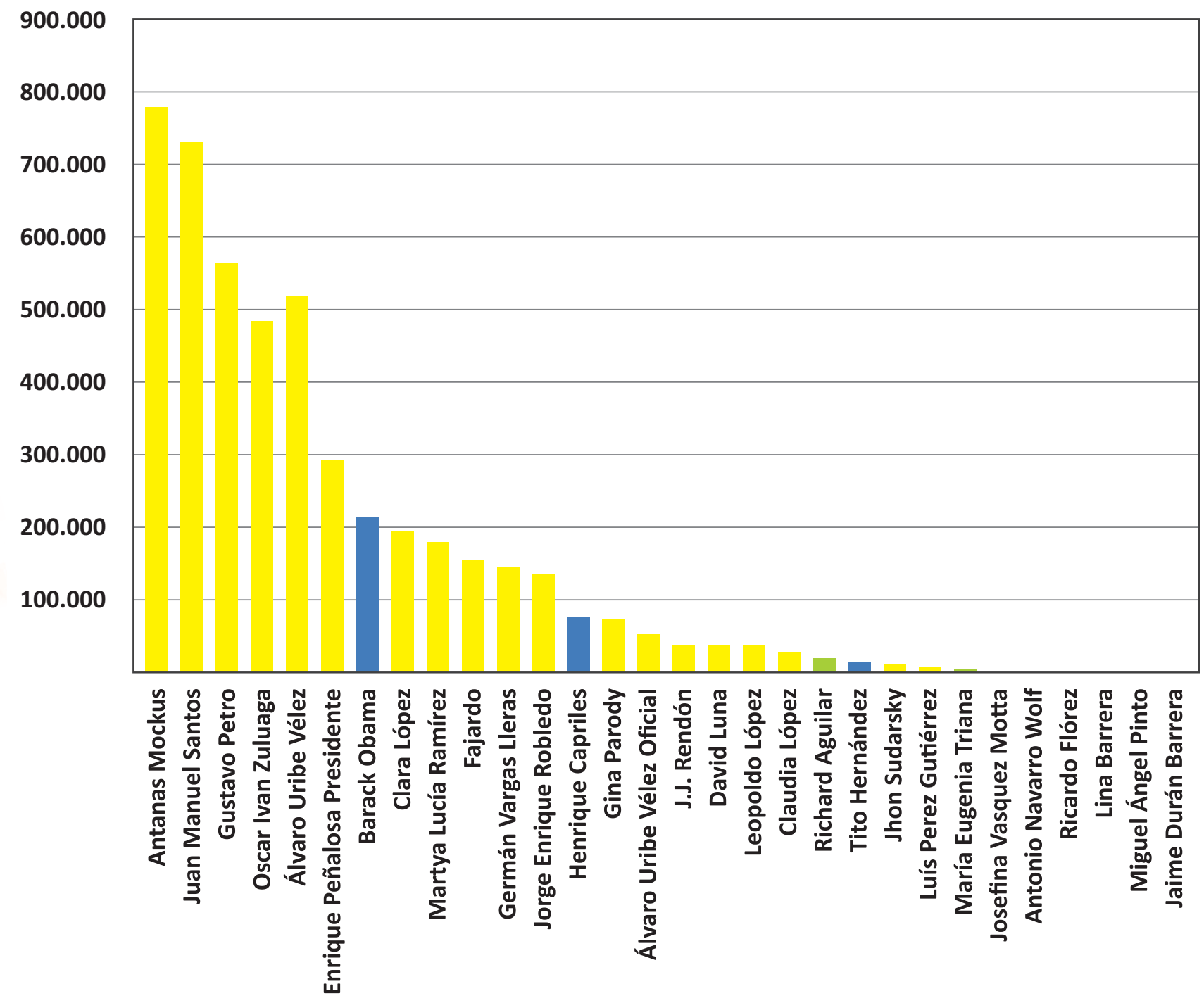

Fuente: El autor.

El manejo del perfil político en Facebook tiene para los candidatos elementos que requieren especial cuidado. Uno de ellos es el nivel de contextualización o descontextualización en que aparecen actividades, informaciones y opiniones, porque representan un riesgo tanto para la privacidad como para la imagen que se quiere mostrar.
Dumortier (2009), explica que la amplia difusión de la información en Facebook a través de amigos que se encuentran en círculos distantes, con una enorme cantidad de gente que no se conoce, está alejada de la lógica natural en la que los seres humanos transmiten información . Por ejemplo, si es de manera oral, requiere de una expectativa racional derivada del interés de 
conocer a la persona referida, por ello puede ocurrir que la información política presentada y compartida se encuentre en contextos dispares, $y$ aunque es posible que los usuarios se sientan a gusto en diferentes contextos, e incluso lleguen a adoptar diferentes personalidades, para la participación política se requiere construir patrones que contribuyan al alineamiento hacia tendencias, ojalá expresadas en los partidos políticos.

En otras palabras, el mercadeo político no debería brindar la posibilidad de compartir la información sin tener en cuenta contextos contradictorios. La manera de mercadear las ideas y actividades políticas en Facebook pareciera ser la de poner información a circular y lograr altos niveles de mensajes compartidos. Valdría la pena preguntarse, ¿en qué contexto un joven votante ve información política y decide opinar o compartirla? ¿Cuando está en la cafetería de la universidad, caminando en un centro comercial, o en la comodidad de su casa? Sería interesante averiguar mucho más sobre este tema.

El punto anterior nos lleva hacia el concepto de la visibilidad en Facebook, que es una cosa diferente a la participación. Si las audiencias son activas, de nada serviría la sola presencia sin participación. Sería una presencia paradójica, sería una presencia ausente de la red, que no representa muchos réditos en el mercadeo político, especialmente desde los jóvenes. Quizá debido a ello es que las experiencias han sido menos exitosas, por ejemplo en España, donde solo el 20,28\% de los 350 diputados tiene presencia en la red, y solamente 9,71\% (34 perfiles) es abierto. No obstante, la estrategia de comunicación es unidireccional, político ciudadano(López \& García, 2011).

Otros puntos que tendrían que tenerse en cuenta como posibles errores son, en primer lugar, el uso no personal del candidato en su perfil. Al ciudadano, y especialmente a los jóvenes, les encantaría interactuar con el político mismo y no con el jefe de prensa, y que por lo menos los mensajes o comentarios sean contestados. Por ejemplo, los hangout de la senadora Claudia López, en donde interactuaba directamente con los posibles electores, probablemente le ayudaron enormemente a sacar una de las votaciones más altas en el país.

En segundo lugar, en Colombia hay más reconocimiento hacia los líderes que hacia los partidos políticos, luego los perfiles deberían apuntar hacia allí su estrategia para cautivar a los jóvenes. Incluso, a largo plazo puede llevar a un mayor alinderamiento político hacia partidos definidos ideológicamente.

En tercer lugar, podría ser un tema de contenido. La mayoría de candidatos colombianos manejan temas en Facebook exaltando sus propias labores políticas, y en muchas ocasiones la hipertextualidad se actualiza con enlaces hacia sus páginas web personales, a noticias en las que son mencionados, o a blogs en los que otros ciudadanos los enaltecen. Es decir, es un contenido basado eminentemente en sus acciones políticas, en los que prevalecen fotografías abrazando niños y sonriendo junto a ancianos, y no en otros temas que son atractivos para las comunidades en Facebook. Probablemente, la popularidad tendría mejor campo si los 
contenidos publicados fueran variados hacia temas ecológicos, humor, enseñanzas, opiniones de otros usuarios que fueran interesantes, etc.

\section{Comentarios}

En cuanto a las posibilidades y las limitaciones que se podrían apreciar en la participación política juvenil desde lo local, existen factores y elementos que deberían ser tenidos en cuenta.

\section{Limitaciones}

Una primera limitación podría ser la característica diferenciadora entre los migrantes digitales, que podrían ser la mayoría de los políticos que dan lineamiento a sus campañas, y las audiencias constituidas por usuarios jóvenes que son nativos digitales. Ello porque existen puntos de vista diferentes entre el diseño y la recepción, entre el contenido expuesto intencionalmente y la respuesta de las audiencias digitales, llamadas en esta época "prosumidores mediáticos", es decir, consumidores que dejan atrás la pasividad para transformarse en productores de contenidos (Mantecon, 2010) que se materializan cuando comparten originales y comentan aportando nuevas ideas en el muro de Facebook. Esta característica requiere una identificación, una segmentación cultural de las audiencias.

Una segunda limitación estribaría en que el crecimiento del conocimiento político y del compromiso a través de las redes sociales no se concreta en la urna. Los usuarios de Facebook pueden conocer mucho sobre partidos, políticos, candidatos, etc., pero es difícil la traducción empírica de toda esta información. A veces es difícil distinguir una estrategia clara entre la participación en el mundo virtual, que en gran medida empieza y termina en él, y la movilización real hacia la participación electoral. Hay una menor disposición hacia la movilización.

Una tercera limitación podría ser que la capacidad de los usuarios para readaptarse a las estrategias políticas o a novedosas formas de compartir en lo virtual, incluyendo redes nuevas, es más rápida que la capacidad de los generadores de estrategias de contenido político y que los mismos candidatos. Quizá sean necesarios profesionales y diseñadores de contenidos capaces de reformar e innovar la manera de llegar a los jóvenes a través del muro de Facebook. Esto incluye, desde luego, nuevas formas de interacción y nuevas dinámicas.

Una cuarta limitación dejaría ver los riesgos de incentivar el uso no democrático de las redes sociales como parte de una estrategia política, como por ejemplo calumniar, desinformar, limitar el contenido, burlarse, etc. El caso de la política colombiana en el último año, durante la campaña presidencial, es el ejemplo claro de lo que no debe hacerse, y contribuye a una menor participación juvenil. Al tedio característico del tema para la juventud, podría sumarse el del agotamiento de no reconocer lo que es verdadero de lo que no, pero además de legitimar el uso de la falsedad como estrategia de campaña. Esta limitación requiere la intervención desde lo jurídico y el amparo legal. 


\section{Posibilidades}

Hay casi once millones de cuentas de Facebook de usuarios jóvenes (entre los 13 y los 24 años) en Colombia. Los usuarios jóvenes que tienen cuentas en Facebook y que pueden votar (18-24 años) son 7.526.720 ciudadanos. Atractivo no solo para cualquier candidato que pensaría en movilizarlos hacia las urnas a partir de los dieciocho años, sino también contribuirían al alineamiento desde los partidos y desde las tendencias políticas que abonan el terreno para fortalecer la democracia. En el área metropolitana de Bucaramanga hay (según datos de owloo.com) un aproximado de 608.000 cuentas de Facebook, cantidad superior a la media nacional con respecto a la población total de habitantes. Colombia (43\%), Bucaramanga $(63 \%)$.

Los patrones contextuales de los usuarios jóvenes de Facebook podrían ser constituidos y visualizados de tal manera que también puedan seguir estableciendo tendencias de alineamientos políticos. Recientemente fue votada en el Congreso de la República una ley para que en Colombia las elecciones se lleven a cabo usando únicamente listas cerradas con el propósito de lograr una mayor identificación de los votantes con las ideas de sus partidos. Quizá pueda lograrse la reversión del proceso de desafiliación colombiano que cada vez se nota menos favorable a partidos y más hacia las personas. Recientes encuestas (Cifras y Conceptos, 2014) revelan que un $42 \%$ de los colombianos no tiene afinidad hacia ningún partido y la más alta es hacia el Partido Liberal (13\%), el Partido de la U (12\%), el Centro Democrático (9\%), el Partido Conservador (6 \%) y el Polo (3\%).
Hay que programar y reprogramar constantemente el contenido político en las redes sociales, siempre con la tendencia de que lleve a una participación efectiva, susceptible de ser medida de acuerdo a las opiniones más que a los simples "like" o la adopción de fans. El que opina tiene la posibilidad de ser reafirmado si está a favor, o de ser convencido si está en contra. Entender que el entusiasmo, las emociones y los miedos juveniles contribuyen al reforzamiento de sus propios argumentos.

La participación no debe ser una simple estrategia de visualización de gente que el usuario no reconocería. Esta debe ser real, que la audiencia sienta que hay alguien al tanto de sus opiniones y que las cosas no se quedan en el aire. Un posible votante en las redes sociales que no encuentra respuesta a sus comentarios por parte del mismo candidato, podría tener mayores probabilidades de deserción o de rechazo.

Si en términos generales la actitud hacia la política de los jóvenes mantiene un carácter negativo, quizá la actitud no debería ser presentar contenido estrictamente político.

El voto por internet y sus características de autonomía y de intemporalidad en el flujo del tiempo, con suficientes garantías de seguridad, podría hacer elevar no solo el voto joven sino también el del resto de población. Esta posibilidad es mejor que el voto obligatorio, pero requiere también de una mayor educación política.

Hay un diálogo más estrecho entre la ciudadanía y el poder político con el uso de las redes sociales. Los casos del llamado "Gobierno 
en Línea" son particularmente interactivos desde Facebook, ello podría constituir una herramienta para canalizar contenido crítico, por ejemplo, desde los líderes de oposición. Uno podría pensar que los políticos locales no tienen mucho interés en el desarrollo de Facebook como herramienta de apalancamiento, porque la manera tradicional en la que se desarrollan las campañas, con reuniones presididas por líderes locales, que muchas veces tienen prácticas conocidas como las cadenas de redes clientelares, la solución de problemas privados a bajo costo como los mercados, la entrega de materiales de construcción, o la misma compra de votos, es bastante efectiva de acuerdo a los recursos económicos que se tengan. No obstante, los nuevos candidatos que elección tras elección desafían los poderes locales y son constantemente derrotados, podrían tener una herramienta valiosa para mover el voto de opinión.

\section{Referencias}

Álvarez, L., Granados, V., \& Hernández, N. (2013). Actitudes hacia la política en estudiantes universitarios. Reflexión Política, 29, 120138.

Alves, R. (2014). ¿Qué implicaciones tiene la revolución digital para el periodismo? Bogotá: VII Encuentro de Periodismo de Investigación. Obtenido de http://www.youtube.com/watch?v=7uZBXyko8 do

Brader, T. (2006). Campaigning for Hearts and Minds, How Emotional Appeals in Political And Work. (U. o. Press, Ed.) Chicago, EUA.
Buckingham, D., \& Rodríguez, C. (2013). Aprendiendo sobre el poder y la ciudadanía en un mundo virtual. Comunicar, 40(20), 4958. Obtenido de Comunicar 40, XX, 4958.: http://www.revistacomunicar.com/index.php?co ntenido $=$ detalles $\&$ numero $=40 \&$ articulo $=40$ 2013-07

Cabalin, C. (2014). Estudiantes conectados y movilizados: el uso de Facebook en las protestas estudiantiles en Chile. Obtenido de Comunicar 43, XXII, 2533.:

http://www.revistacomunicar.com/index.php?co ntenido $=$ detalles \& numero $=43 \&$ articulo $=43$ 2014-02

Castells, M. (2009). Comunicación y Poder. Madrid, España: Alianza Editorial.

Cifras y Conceptos. (2014). Obtenido de Polimétrica. Análisis sobre instituciones, economía, política y sociedad.: http://www.cifrasyconceptos.com/images/desca rgas/polimetrica/Polimetrica_resultadosEvaluac in_22072014_PAZMINISTROS.pdf

Dallmeier, S. (Febrero de 2014). Seminario Internacional de Marketing Político. Universidad Central. Bogotá, Colombia: Universidad Central.

Duch, J. (2011). ¿Votos por internet para aumentar la participación electoral? Obtenido de Servicio de Prensa del Parlamento Europeo: http://www.europarl.europa.eu/news/es/newsroom/content $/ 20110321 \mathrm{STO} 15986 / \mathrm{html} / \% \mathrm{C} 2 \%$ BFVotos-por-internet-para-aumentar-laparticipaci $\% \mathrm{C} 3 \% \mathrm{~B} 3 n$-electoral

Dumortier, F. (2009). Facebook y los riesgos 
de la descontextualización de la información. Revista de Internet, Derecho Y Política. Universidad Abierta de Catalunya, 9, 3244. Obtenido de

http://journals.uoc.edu/index.php/idp/article/vie w/n9_dumortier/n9_dumortier_esp

Fidalgo, A., Serrano, A., Cnhavilas, J., \& Correia, J. (2013). El ser humano como portal de comunicación: la construcción del perfil en el teléfono móvil. Revista Latina de Comunicación Social., 68, 545565. doi:doi:10.4185/RLCS2013-988

Flores, J. (2009). Nuevos modelos de comunicación, perfiles y tendencias en las redes sociales. Comunicar, 16(33), 73-81. doi:doi:10.3916/c33-2009-02-007

García, M., Del Hoyo, M., \& Fernández, C. (2014). Jóvenes comprometidos en la red: el papel de las redes sociales en la participación social activa. Comunicar, 43, 3543. Obtenido de http://www.revistacomunicar.com/verpdf.php?n umero=43\&articulo=43-2014-03.

Habermas, J. (1999). Teoría de la acción comunicativa. Racionalidad de la acción y racionalización social. Madrid, España: Taurus.

Leonard, L. (01 de Octubre de 2012). Youth Participation in Civic Engagement Through Social Media: a case study. Recuperado el 25 de $\mathrm{S}$ e p t i e m b r e d e $2014, \quad d$ e http://spir.aoir.org/index.php/spir/article/view/2 3

López, M., \& García, J. (2011). Marketing político, gobierno y redes sociales: ¿difundir, informar, comunicar, relacionarse? Obtenido de
II Congreso Internacional Latina de Comunicación Social. Tenerife: Universidad de la Laguna: http://www.ull.es/publicaciones/latina/10SLCS/ actas_2010/001MiguelTunez02.htm

Mantecon, A. (2010). Del público al prosumidor. Nuevos retos para los estudios de consumo cultural. Entretextos, 6, 3742. Obtenido de

http://www.scielo.org.mx/scielo.php?pid=S018 8-70172012000200001\&script $=$ sci_arttext

Mayo, F. (2005). Contenidos y prácticas políticas de los internautas en el Estado Español. Análisis diferencial de internautas y no internautas en su comportamiento político y asociativo a fecha de Abril del 2002. Obtenido de http://www.uoc.edu/in3/dt/esp/mayo0505.html

Mires, F. (2009). La revolución que nadie soñó o la otra posmodernidad: la revolución microelectrónica, la revolución feminista, la revolución ecológica, la revolución política, la revolución paradigmática. (3rd ed ed.). Bogotá: Promolibro.

Orihuela, L. (2008). Internet: la hora de las redes sociales. Nueva Revista, 119, 5762. Obtenido de http://www.ecuaderno.com/2008/10/14/la-horade-las-redes-sociales/

Pérez, C. (2006). Enfoques teórico metodológico en el estudio de la participación electoral. Cuestiones políticas No.37. (F. d. LUZ., Ed.)

Poushter, J. (2014). In India, few use social media to share political views. Recuperado el 24 
d e Septiembre de 2014, d e http://www.pewresearch.org/fact tank/2014/04/08/in-india-few-use-social-mediato-share-political-views/

Prensky, M. (2010). Nativos e Inmigrantes Digitales. Madrid: SEK. Obtenido de http://www.marcprensky.com/writing/PrenskyNATIVOS E INMIGRANTES DIGITALES (SEK).pdf

Reniun, J. (2008). Ocho dudas razonables sobre la necesidad del voto electrónico. Revista de Internet, Derecho Y Política. Univerisidad
Abierta de Catalunya., 6, 3244. Obtenido de http://www.uoc.edu/idp/6/dt/esp/reniu.pdf

Sanchez, C. (2013). Foro sobre el papel del ciudadano en el fortalecimiento de la $\mathrm{d}$ e m o c r a c i a. O b t e n i d o d e http://www.noticiasrptv.com/site/vernoticia.asp ?ac $=$ Solo $\% 20$ el $\% 2010 \% \% 20 \mathrm{de} \% 201$ os $\% 20 \mathrm{j} \%$ C3\%B3venes\%20votan\%20en\%20el\%20pa\%C 3\%ADs. $\% 20 \&$ WPLACA $=503$

Westen, D. (2007). The Political Brain, The Role of Emotion in Deciding the Fate of the Nation. Nueva York: Public Affairs. 